\title{
Elektrikli Araçlarda Batarya Şarj Ünitesi için LLC Rezonans Dönüştürücünün Kurulumu, Modellemesi ve Analizi \\ Ünal YILMAZ*,a $\mathbb{D}$, Ali KIRÇAY ${ }^{\mathrm{b},}$ \\ ${ }^{a,{ }^{*}}$ Harran Üniversitesi Elektrik Elektronik Mühendisliği Bölümü, 63000, Şanlıurfa, Türkiye
}

\begin{tabular}{l} 
MAKALE \\
BİLGISI \\
\hline \\
Alınma: 30.03 .2021 \\
Kabul: 30.11 .2021 \\
\hline \\
Anahtar Kelimeler: \\
Elektrikli Araçlar, \\
Batarya Şarj Ünitesi, \\
LLC Rezonans \\
Dönüştürücü
\end{tabular}

\section{öz}

Bu çalışmada, elektrikli araçların batarya şarj ünitelerine uyumlu bir yarım köprü yalıtımlı LLC rezonans dönüştürücü modellemesi, kurulumu ve analizi yapılmıştır. Önerilen çalışmanın laboratuvar ortamında kurulumu için F28027 DSP tabanlı kontrol kartı ve 'TMDSHVRESLLCKIT' geliştirme kiti kullanılmıştır. Ayrıca, önerilen çalışmanın analiz aşaması için, $300 \mathrm{~W}$ gücünde, $380 \mathrm{~V}$ giriş gerilimi ve $12 \mathrm{~V}$ çıkış geriliminde bir LLC rezonans dönüştürücü PI tabanlı kontrol yöntemi ile modellenmiştir. Analizi yapılan çalışma, ani değişen giriş gerilimi $(380 \mathrm{~V}-400 \mathrm{~V})$ ve 4 farklı yük durumunda $(\% 100, \% 75, \% 50, \% 25)$ incelenmiştir. Analiz çalışmasının benzetim ortamında gerçekleştirilmesi için Matlab/Simulink programı kullanılmıştır.

\section{Installation, Modeling and Analysis of LLC Resonance Converter for Battery Charger in Electric Vehicles}

\section{ARTICLE \\ INFO}

Received: 30.03 .2021

Accepted: 30.11 .2021

Keywords:

Electric Vehicles,

Battery Charger, LLC

Resonance Converter

\begin{abstract}
In this study, a half-bridge isolated LLC resonance converter modeling, installation and analysis is performed, which is compatible with the battery charging units of electric vehicles. F28027 DSP-based control card and 'TMDSHVRESLLCKIT' development kit were used for the setup of the proposed study in the laboratory environment. In addition, for the analysis phase of the proposed study, an LLC resonance converter with $300 \mathrm{~W}$ power, $380 \mathrm{~V}$ input voltage and $12 \mathrm{~V}$ output voltage was modeled with PI-based control method. The analyzed study has been investigated in case of suddenly changing input voltage (380 V-400 V) and 4 different load conditions $(100 \%, 75 \%, 50 \%, 25 \%)$. Matlab / Simulink program was used to carry out the analysis study in a simulation program.
\end{abstract}

https://dx.doi.org/10.30855/gmbd.2021.03.12

\begin{tabular}{|l|l|l|l|}
\hline \multicolumn{4}{|c|}{ Kisaltmalar ve Semboller } \\
\hline EA & Elektrikli Araç & $R e$ & AC eşdeğer yük \\
\hline BŞÜ & Batarya Şarj ünitesi & $R o$ & Yük \\
\hline PFC (Power Factor Correction) & Güç faktörü düzeltmesi & Vin & Giriş Gerilimi \\
\hline EMI (Electromagnetic Interference) & Elektromanyetik Girişim & Lm & Manyetik Bobin \\
\hline PWM (Pulse Width Modulation) & Darbe Genişlik Modülasyonu & $Q e$ & Kalite Faktörü \\
\hline AC & Alternatif Akım & Ln & Bobin katsayı \\
\hline DC & Doğru Akım & $f p$ & Düşük Frekans Sınırı \\
\hline$L r$ & Rezonans Bobini & $P$ & Güç \\
\hline$C r$ & Rezonans Kapasitör & Vo & Ç1kış gerilimi \\
\hline$F s w$ & Anahtarlama Frekansı & fco & Rezonans Frekans1 \\
\hline
\end{tabular}

*Sorumlu yazar: uyilmaz@harran.edu.tr

To cite this article: Ü. Yılmaz and A. Kıryaç, "Installation, Modeling and Analysis of LLC Resonance Converter for Battery Charger in Electric Vehicles", Gazi Journal of Engineering Sciences, vol.7, no.3, pp. 298-308, 2021.

doi:10.30855/gmbd.2021.03.12 


\section{GİRIŞ̧ (INTRODUCTION)}

Elektrikli araçlarda batarya şarj üniteleri literatürde dâhili (On-board) ve harici (Off-board) olmak üzere iki farklı başlık altında incelenmektedir. Harici batarya şarj üniteleri bataryayı şarj etmek için dc akımını kullanır, bu da şarj istasyonunda ac-dc dönüştürme işleminin gerçekleştiği anlamına gelir. Harici batarya şarj ünitelerinin en önemli avantajları, yüksek güç yoğunluğudur, bu da şarj süresini önemli ölçüde kısaltır ve batarya şarj ünitesi arabanın içinde olmadığ 1 için yer tasarrufu sağlar. Bununla birlikte, karmaşı batarya yönetim sistemi, bataryada aşırı ısınma sorunu ve yüksek güç gereksinimi gibi önemli dezavantajlar vardır. Bu zorluklar nedeniyle, EA'lar için off-board batarya şarj üniteleri genellikle tercih edilmemektedir [1]. Diğer taraftan, dâhili batarya şarj üniteleri (BŞÜ) EA'ların ev ve ofislerde mevcut standart bir prizi kullanarak bataryayı şarj etmelerine izin verir. BŞÜ'lerin ana parçaları; Şekil 1 (b) 'de detaylıca gösterilmektedir.

Dâhili batarya şarj üniteleri, tek kademeli ve iki kademeli olmak üzere iki farklı topoloji olarak incelenmektedir. Tek kademeli batarya şarj üniteleri, kolay uygulanabilirlik, düşük gerilim kademesinde çalışırken yüksek etkinliğe sahip olmaları, düşük uygulama maliyeti ve yüksek verimlilik (düşük dönüştürme aşaması) gibi bazı avantajlara sahiptir. Ancak, tek kademeli batarya şarj cihazları, düşük güç $(\leq 1 \mathrm{~kW})$ uygulamaları için uygundur ve bu topolojinin önemli dezavantajlarından biri, gerilim dalgalanmasını en aza indirmek için büyük bir çıkış elektrolit kapasitörü gerektirir [2-8].Bir diğer dâhili batarya şarj ünitesi modeli ise iki kademeli batarya şarj ünitesidir. İyi tasarlanmış iki kademeli batarya şarj üniteleri; yüksek güç yoğunluğu, düşük harmonik, yüksek verimlilik ve yüksek güç faktörü gibi ciddi avantajlara sahip iken yüksek maliyet ve karmaşık kontrol yöntemleri gibi dez avantajları da mevcuttur.

İki kademeli batarya şarj ünitelerinin ilk kademesi, güç faktörü düzenleyicisi (PFC) ile birlikte performans gösteren bir ac-dc dönüştürücü devresi iken ikinci kademesi ise, galvanik yalıtımlı dc-dc dönüştürücülerdir. İki kademeli BŞÜ'lerin, güç yoğunluğu, verimlilik, güvenilirlik ve yaşam süresi gibi bazı özelliklerini iyileştirmek ve performansını artırmak için dc-dc dönüştürücülerin eksik yönlerinin iyileştirilmesi önemli bir rol oynar [9], [10], [11].

LLC rezonans dönüștürücüler, yüksek güç yoğunluğu, yüksek verimlilik ve maliyet etkinliği yönünden avantaj sağladığından dolayı, farklı endüstriyel uygulamalarda yaygın olarak tercih edilmektedir. Ayrica, LLC rezeonans dönüştürücülerde anahtarlama elemanı (MOSFET)'lerin açılıp kapanma işlemlerinde yumuşak anahtarlamaya olanak sağladığından dolayı anahtarlama kayıpları oldukça düşüktür.

$\mathrm{Bu}$ çalışmada Elektrikli araçların batarya şarj ünitelerinde kullanılabilen bir yarım köprü LLC rezonans dönüştürücü labarotuvar ortamında ve benzetim programında kurulup analiz edilmiştir. Ayrıca kolay uygulanabilir, verimlilik ve güvenilirlik gibi önemli avantajlara sahip olduğundan dolayı bu çalışmada çıkış gerilim kontrolü için PI kontrol yöntemi tercih edilmiştir [12].

Önceki çalışmalar incelendiğinde, [13]'te hibrit elektrikli araçların batarya şarj ünitesi için bir LLC rezonans dönüştürücü tasarlanmış ve geleneksel yöntemlere göre temel harmonik yaklaşımlarının sebep olduğu hataları azaltmak için çalışma modu düzenlemesi gerçekleştirilmiştir. Önerilen çalışma için $3.3 \mathrm{~kW}$ 'lık bir örnek devre adım adım tasarlanmış ve maksimum verimlilik \%98.2 olarak bulunmuştur.

[14]'te, sıfıra yakın çıkış gerilimi, sıfır çıkış akımından maksimum çıkış gücüne kadar, bataryanın akım-gerilim düzlemindeki neredeyse tüm bölgeleri kapsayabilen çok yönlü bir L3C2 rezonans dönüştürücü önerilmiştir. Önerilen çalışmanın doğruluğunu kanıtlamak için $950 \mathrm{~W}$ gücünde bir örnek devre modellenmiş ve maksimum \%96 verimliliğe ulaştığı belirtilmiştir.

[15]'te elektrikli araçlarda batarya şarj ünitesi için için paralel iki transformatörlü yeni bir LLC rezonans dönüştürücü önerilmiştir. Önerilen topolojide, şarj süresi boyunca sıfır gerilim anahtarlamasına olanak sağladığı için anahtarlama kayıpları azaltılmıştır. Ek olarak, önerilen çalışmada, batarya sistemi için geniş bir çıkış gerilim aralığı sağlanmıştır. Önerilen çalışmanın doğruluğunu desteklemek için $700 \mathrm{~W}$ gücünde bir örnek devre oluşturulmuştur. Önerilen çalışmada yaklaşık \%93.6 verimliliğe ulaştı̆̆ belirtilmiştir.

Referans [16]'da hafif elektrikli araçların batarya şarj ünitesi için yumuşak anahtarlama yapabilen LLC rezonans dönüştürücü ve şarj işlemi için yeni bir kontrol algoritması önerilmiştir. 


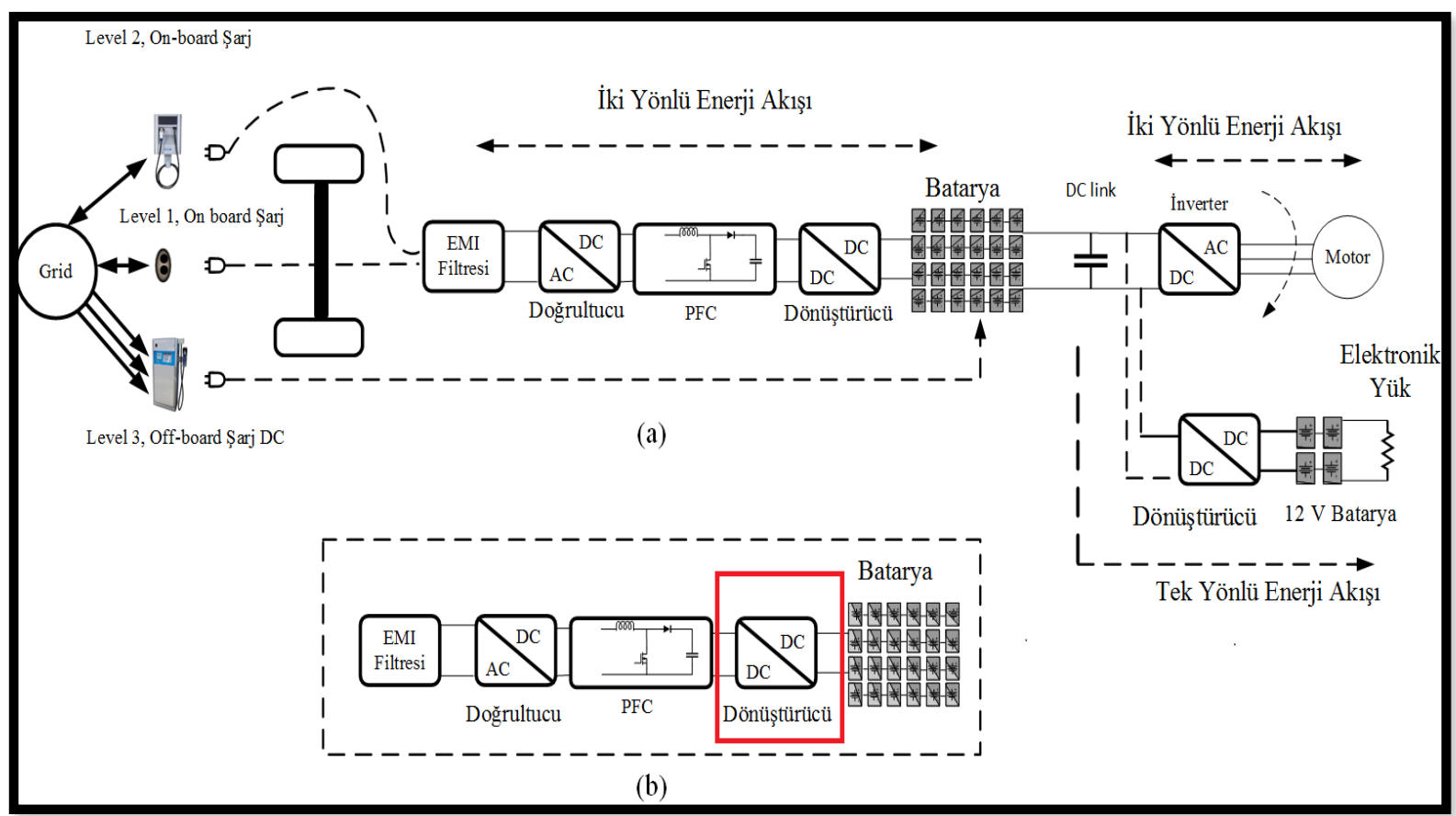

Şekil 1. EA'lar için Genel Güç Koşullandırma Ünitesi (a), On-board batarya şarj ünitesi (b). (General Power Conditioning Unit (a), On-board battery charger (b) for EVs.)

Önerilen çalışmanın doğruluğunu desteklemek için 1 ve $1.5 \mathrm{~kW}$ gücünde devre modellemesi ve deneysel analizi yapılmış ve sonuçta yaklaşık \%96.4 oranında bir verimlilik elde edilmiştir. Tablo 1 de önceki çalışmaların detaylı elektriksel özellikleri sunulmuştur.

Tablo 1. Literatürdeki çalışmaların elektriksel özellikleri (Electrical characteristics of studies in the literature)

\begin{tabular}{cccccc}
\hline Ref. & Güç (W) & Giriş Gerilimi (V) & Çıkış Gerilimi (V) & \multicolumn{1}{c}{ Verim (n) } & Anah. Frekansı \\
\hline$[10]$ & $3.1 \mathrm{~kW}$ & $400 \mathrm{~V}$ & $120 \mathrm{~V}$ & $96.2 \%$ & $85-100 \mathrm{kHz}$ \\
\hline$[11]$ & $3.1 \mathrm{~kW}$ & $400-120 \mathrm{~V}$ & $120-24 \mathrm{~V}$ & $95.3 \%$ & $85 \mathrm{~Hz}$ \\
\hline$[13]$ & $3.3 \mathrm{~kW}$ & $390 \mathrm{~V}-420 \mathrm{~V}$ & $250 \mathrm{~V}-450 \mathrm{~V}$ & $98.2 \%$ & $150 \mathrm{kHz}$ \\
\hline$[14]$ & $950 \mathrm{~W}$ & $400 \mathrm{~V}$ & $96 \mathrm{~V}$ & $96 \%$ & $145 \mathrm{kHz}$ \\
\hline$[15]$ & $700 \mathrm{~W}$ & $400 \mathrm{~V}$ & $36 \mathrm{~V}-58 \mathrm{~V}$ & $93.6 \%$ & $100 \mathrm{kHz}$ \\
\hline$[16]$ & $1-1.5 \mathrm{~kW}$ & $250 \mathrm{~V}-310 \mathrm{~V}$ & $25.6 \mathrm{~V}-33.6 \mathrm{~V}$ & $96.4 \%$ & $30-100 \mathrm{kHz}$ \\
\hline$[17]$ & $2 \mathrm{~kW}$ & --- & --- & $95 \%$ & --- \\
\hline$[18]$ & $345 \mathrm{~W}$ & $390 \mathrm{~V}$ & $57.6 \mathrm{~V}$ & $96.3 \%$ & $150 \mathrm{kHz}$ \\
\hline$[19]$ & $500 \mathrm{~W}$ & $330 \mathrm{~V}$ & $12 \mathrm{~V}$ & --- & $200 \mathrm{kHz}$ \\
\hline$[20]$ & $7.5 \mathrm{~kW}$ & $340 \mathrm{~V}-360 \mathrm{~V}$ & $150 \mathrm{~V}-500 \mathrm{~V}$ & $98 \%$ & $115 \mathrm{kHz}$ \\
\hline$[21]$ & ---- & $400 \mathrm{~V}$ & $350 \mathrm{~V}$ & ---- & $100 \mathrm{kHz}$ \\
\hline
\end{tabular}

Elektrikli araçlarda tüm güç devreleri bataryalardan beslendiği için batarya teknolojisi elektrikli araçlar için hayati öneme sahiptir.
Elektrikli araçlarda kullanılan batarya çeşitleri ve genel özellikleri Tablo 2'de sunulmuştur [22]. 
Tablo 2. Elektrikli Araçlarda Kullanılan Batarya Çeşitleri (Types of Batteries Used in Electric Vehicles) [22]

\begin{tabular}{ccccc}
\hline Batarya Çeşitleri & $\begin{array}{c}\text { Nominal } \\
\text { Gerilim }(\mathbf{V})\end{array}$ & $\begin{array}{c}\text { Enerji } \\
\text { Yoğunlu } \\
(\mathbf{W h} / \mathbf{k g})\end{array}$ & $\begin{array}{c}\text { Çalışma } \\
\text { Sıcaklı̆̆ } \\
\mathbf{( \mathbf { o }} \mathbf{C})\end{array}$ & $\begin{array}{c}\text { Üretim Maliyeti } \\
\mathbf{( \$ / k W h )}\end{array}$ \\
\hline Kurşun asit (Pb-Acid) & 2 & 35 & $-15 \leq \mathrm{T} \leq+50$ & 60 \\
\hline Nikel Kadmiyum (Ni-Cd) & 1.2 & $50-80$ & $-20 \leq \mathrm{T} \leq+50$ & $250-300$ \\
\hline Nikel Metal & 1.2 & $70-95$ & $-20 \leq \mathrm{T} \leq+60$ & $200-250$ \\
\hline Zebra (Ni-Mh) & 2.6 & $90-120$ & $+245 \leq \mathrm{T} \leq+350$ & $230-345$ \\
\hline Lityum-İon (Li-ion) & 3.6 & $118-250$ & $-20 \leq \mathrm{T} \leq+60$ & 150 \\
\hline Lityum-İon Polimer (Li-Po) & 3.7 & $130-225$ & $-20 \leq \mathrm{T} \leq+60$ & 150 \\
\hline Lityum-İon Fosfat (LiFePO4) & 3.2 & 120 & $-45 \leq \mathrm{T} \leq+70$ & 350 \\
\hline Çinko-Hava (Zn-Air) & 1.65 & 460 & $-10 \leq \mathrm{T} \leq+55$ & $90-120$ \\
\hline Lityum-Sülfür (Li-S) & 2.5 & $350-650$ & $-60 \leq \mathrm{T} \leq+60$ & $100-150$ \\
\hline Lityum-hava (Li-air) & 2.9 & $1300-2000$ & $-10 \leq \mathrm{T} \leq+70$ & ---- \\
\hline
\end{tabular}

\section{MATERYAL VE METOT (MATERIAL AND METHOD)}

LLC rezonans dönüştürücü, yüksek verimliliği, düşük elektromanyetik paraziti (EMI), geniş yük gerilimi çalışma aralığı ve ayrıca yüksek güç yoğunluğu altında performans gösterme yeteneği ile dikkat çekici bir yalıtımlı devre modeli haline gelmiştir. Fakat, LLC rezonans dönüştürücülerinin yüksek frekanslı işlemlerde bazı sorunları vardır, bunlar parazittik kapasitans ve kaçak endüktansa daha yüksek duyarlılık, anahtarlama cihazlarında daha yüksek stres ve artan EMI'dir. Yarım köprü LLC rezonans dönüştürücünün devre yapısı Şekil 2 'de gösterilmiştir [23-26].

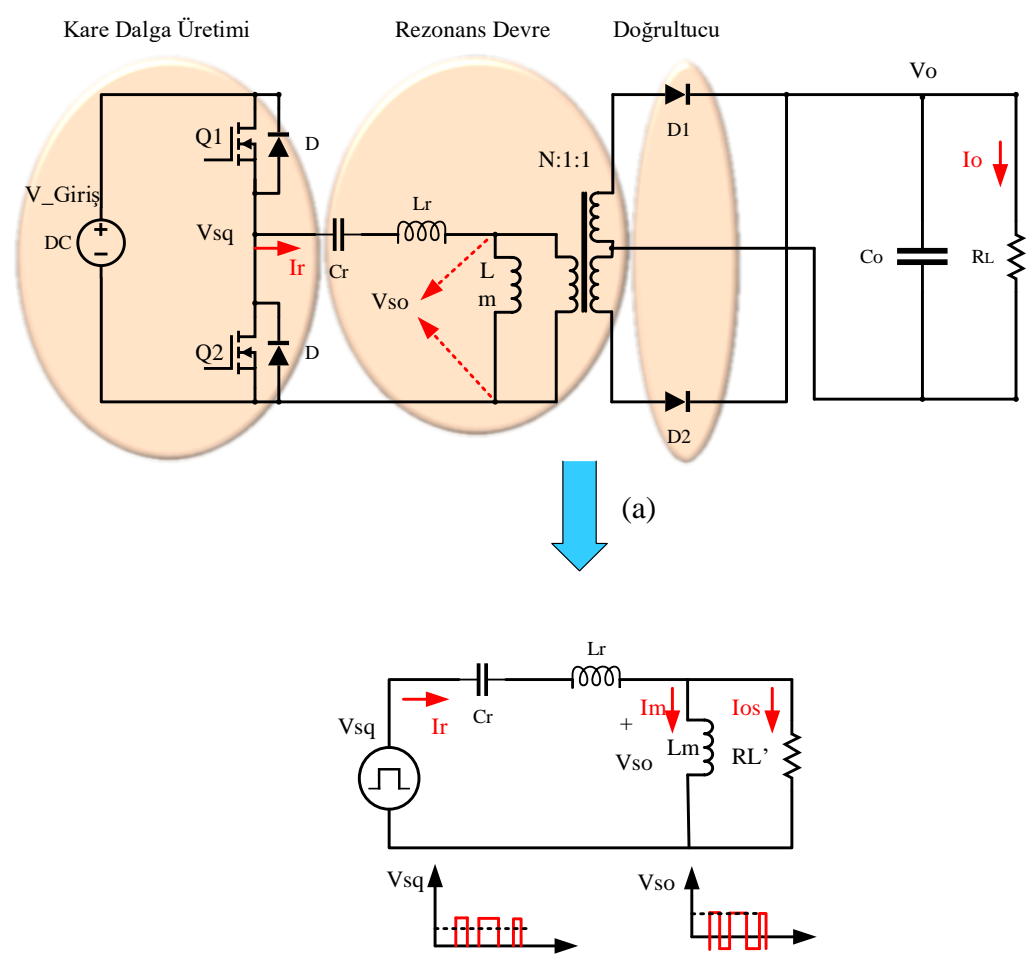

(b)

Şekil 2. LLC rezonans dönüştürücü (a), AC eşdeğer devre (b) (LLC resonant Converter (a), AC equivalent circuit (b))

Yarım köprü LLC seri Rezonans dönüştürücü tasarımı için aşağıdaki eşitlikler kullanılmıştır. Anahtarlama frekansının eşitliği aşağıdaki gibidir.

$$
\mathrm{f}_{0}=\frac{1}{2 \pi \sqrt{\mathrm{L}_{\mathrm{r}} \mathrm{C}_{\mathrm{r}}}}
$$


Çıkış gerilimini kontrol edebilmek için frekans kontrolü gerekir bundan dolayı tepe rezonans $\left(f_{c o}\right)$ değeri rezonans frekansına eşit olmalıdır. Manyetik indüktans $L_{m}$ devreye eklenince tepe rezonans frekansı yük değerinin bir fonksiyonu haline gelir. Yük değişimi frekans değerini etkileyeceğinden dolayı bu durumda rezonans frekans1 $f_{p} \leq f_{0} \leq f_{c o}$ olmalıdır. Kutup frekansının eşitliği aşağıdaki gibidir.

$$
f_{p}=\frac{1}{2 \pi \sqrt{\left(L_{r}+L_{m}\right) C_{r}}}
$$

Bu durumda devre yüksüz ise $f_{p}=f_{c o}$ olur. Yük $\operatorname{arttıkça~} f_{\text {co }}$ yavaş yavaş $f_{o}$ 'a yaklaşır ve kısa devre durumunda ise $f_{c o}=f_{0}$ olur [23]. Giriş kare dalga geriliminin temel harmonik eşitliği aşağıdaki gibidir.

$$
\mathrm{v}_{\mathrm{ge}}(\mathrm{t})=\frac{2}{\pi} * \mathrm{~V}_{\mathrm{DC}} * \sin \left(2 \pi \mathrm{f}_{\mathrm{sw}} \mathrm{t}\right)
$$

Etkin değerinin ise eşitliği aşağıdaki gibidir.

$$
\mathrm{V}_{\mathrm{ge}}=\frac{2}{\pi} * \mathrm{~V}_{\mathrm{DC}}
$$

Çıkış tarafında ise kare dalganın temel gerilim eşitliği aşağıdaki gibidir.

$$
v_{o e}(t)=\frac{4}{\pi} \cdot n \cdot V_{O} \cdot \sin \left(2 \pi f_{s w} t-\theta_{V}\right)
$$

$\Theta_{v}, V_{g e}$ ve $V_{o e}$ arasındaki faz açısıdır. Çıkış geriliminin etkin değerinin eşitliği aşağıdaki gibidir [23].

$$
\mathrm{V}_{\mathrm{oe}}=\frac{2 \sqrt{2}}{\pi} * \mathrm{n} * \mathrm{~V}_{\mathrm{o}}
$$

$\mathrm{V}_{\mathrm{oe}}$ değerine karşılık gelen akım değerinin $\left(\mathrm{I}_{\mathrm{oe}}\right)$ eşitliği aşağıdaki gibidir.

$$
\mathrm{I}_{\mathrm{oe}}(\mathrm{t})=\frac{2}{\pi} * \frac{1}{\mathrm{n}} * \mathrm{I}_{\mathrm{O}} * \sin \left(2 \pi \mathrm{f}_{\mathrm{sw}} \mathrm{t}-\theta_{\mathrm{i}}\right)
$$

$\Theta_{i}, V_{o e}$ ve $I_{o e}$ arasındaki faz açısıdır. Çıkış akımının etkin değerinin eşitliği aşağıdaki gibidir.

$$
\mathrm{I}_{\mathrm{oe}}=\frac{\pi}{2 \sqrt{2}} * \frac{1}{n} * \mathrm{I}_{\mathrm{o}}
$$

Yükün AC eşdeğerinin eşitliği aşağıdaki gibidir.

$$
R_{e}=\frac{V_{o e}}{I_{o e}}=\frac{8 * n^{2}}{\pi^{2}} \frac{V_{o}}{I_{o}}=\frac{8 * n^{2}}{\pi^{2}} R_{L}
$$

Gerilim kazancının eşitliği aşağıdaki gibidir.

$$
\begin{aligned}
& M_{g}=\frac{V_{o e}}{V_{g e}} \\
& =\left|\frac{J X_{L m} / / R_{e}}{\left(J X_{L m} / / R_{e}\right)+j\left(X_{L r}-X_{C r}\right)}\right| \\
& =\left|\frac{J w L_{m} / / R_{e}}{\left(J w L_{m} / / R_{e}\right)+J w L_{r}+\frac{1}{J w C_{r}}}\right|
\end{aligned}
$$

Son durumda çıkış geriliminin eşitliği aşağıdaki gibidir.

$$
\mathrm{V}_{\mathrm{o}}=M_{g} * \frac{1}{n} * \frac{V_{\text {in }}}{2}
$$

Giriş kısmında ise iki bobin değeri, tek bir bobin gibi düşünülülür ve eşitliği aşağıdaki gibidir.

$$
L_{n}=\frac{L_{m}}{L_{r}}
$$

Kalite faktörünün eşitliği aşağıdaki gibidir.

$$
\mathrm{Q}_{\mathrm{e}}=\frac{\sqrt{L_{r} / C_{r}}}{R_{e}}
$$

Eşitliklerde kullanılan ifadeler açıklanacak olursa; $L r$ rezonans bobini, $L m$ manyetik bobin, $C r$ rezonans kapasitörü, $M g$ gerilim kazancı, $V_{D C}$ giriş gerilimi, $V o$ çıkış gerilimi, Io çıkış akımı, $n$ trafo dönüştürüme oranı, $f s w$ anahtarlama frekans1 [23].

\subsection{LLC Rezonans Dönüștürücü devresinin} Laboratuvar Ortamında Kurulumu (Installation of the LLC Resonant Converter circuit in a Laboratory Environment)

LLC Rezonans dönüştürücünün laboratuvar ortamında kurulması Şekil 3'te detaylıca gösterilmiştir. Deney seti Değişken DC güç kaynağı, 0-10 ohm 30 Ampere kadar dayanıklı Değişken yük, Multimetre, Yüksek gerilimli LLC Rezonans devresi, program çalıştırmak ve kontrol etmek için bir adet bilgisayardan oluşmuştur. 


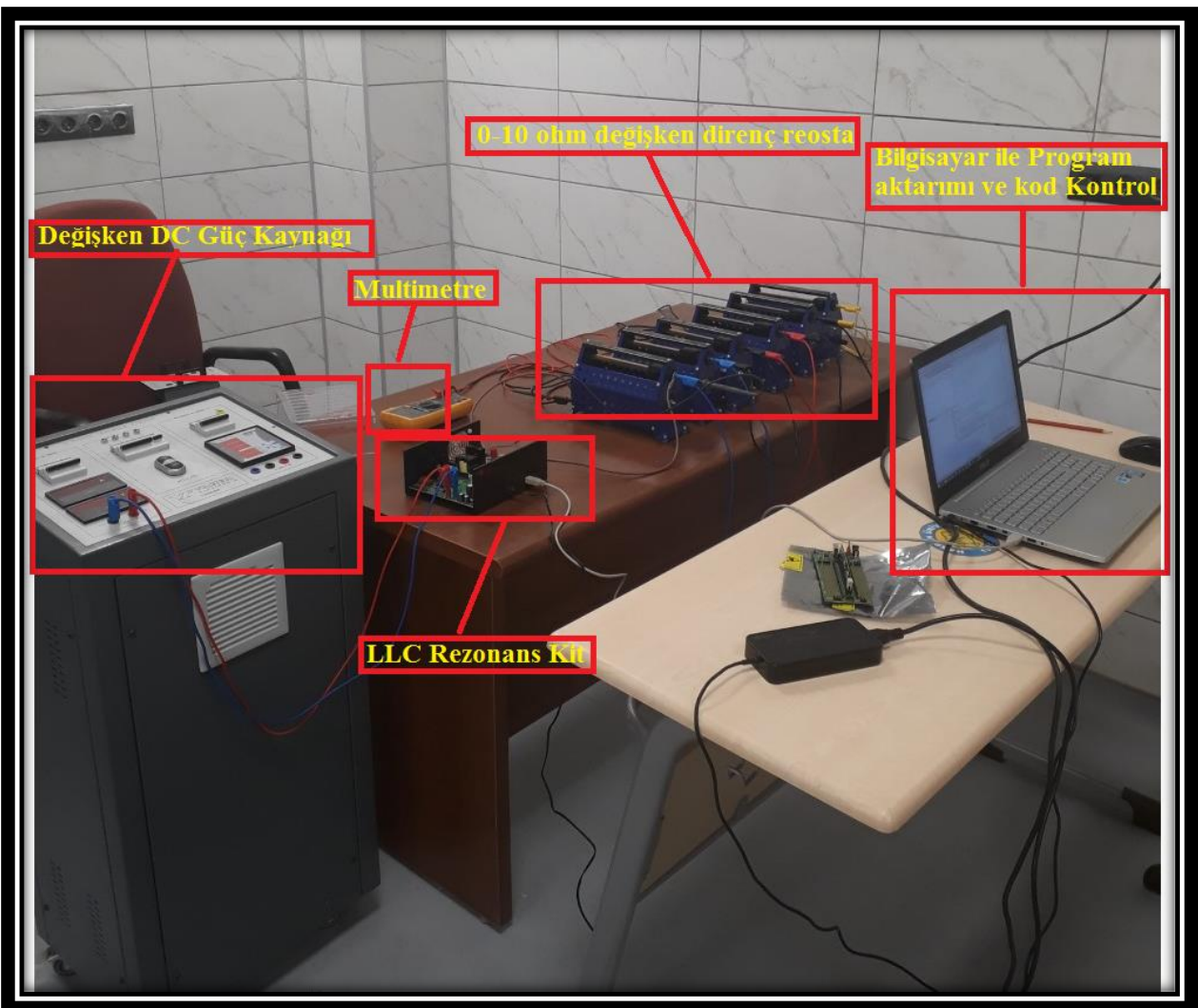

Şekil 3. LLC Rezonans Dönüştürücü Devresi ve deney setinin Laboratuvar ortamında Kurulumu (Installation of LLC Resonant Converter Circuit and Experiment Set in Laboratory Environment)

\subsection{LLC Rezonans Dönüştürücü devresinin} Benzetim Ortamında Kurulması (Setting up the LLC Resonant Converter circuit in a Simulated Environment)

Yarım köprü LLC Rezonans Dönüştürücünün Matlab/Simulink benzetim ortamındaki kurulumu
Şekil 4'te gösterilmiştir. Tasarımı yapılan yarım köprü seri LLC rezonans dönüştürücünün tüm elektriksel özellikleri Tablo 3 'te gösterilmiştir.

Tablo 3. LLC Rezonans Dönüştürücünün Elektriksel Özellikleri (Electrical Characteristics of LLC Resonant Transducer)

\begin{tabular}{|c|c|c|c|c|c|}
\hline Ísim & Sembol & Değer & $\dot{I s i m}$ & Sembol & Değer \\
\hline Rezonans Bobini & $L r$ & $76.186 \mu \mathrm{H}$ & Manyetik Bobin & $L m$ & $207.779 \mu \mathrm{H}$ \\
\hline Rezonans Kapasitör & $\mathrm{Cr}$ & $33.248 \mathrm{nF}$ & Kalite Faktörü & $Q e$ & 0.4 \\
\hline Anahtarlama Frekansl & $F s w$ & $100 \mathrm{kHz}$ & Bobin katsayl & $L n$ & 5 \\
\hline AC eşdeğer yük & $R e$ & 119.67 & Düşük Frekans Sinırı & $F p$ & $55.277 \mathrm{kHz}$ \\
\hline$Y \ddot{u} k$ & Ro & $0.48 \Omega$ & Güç & $P$ & $300 \mathrm{~W}$ \\
\hline Giriş Gerilimi & Vin & $380 \mathrm{~V}$ & Çıkış gerilimi & Vo & $12 \mathrm{~V}$ \\
\hline Oransal Kazanç & $K p$ & 2.283 & Integral Kazanç & $K i$ & 7.623 \\
\hline
\end{tabular}




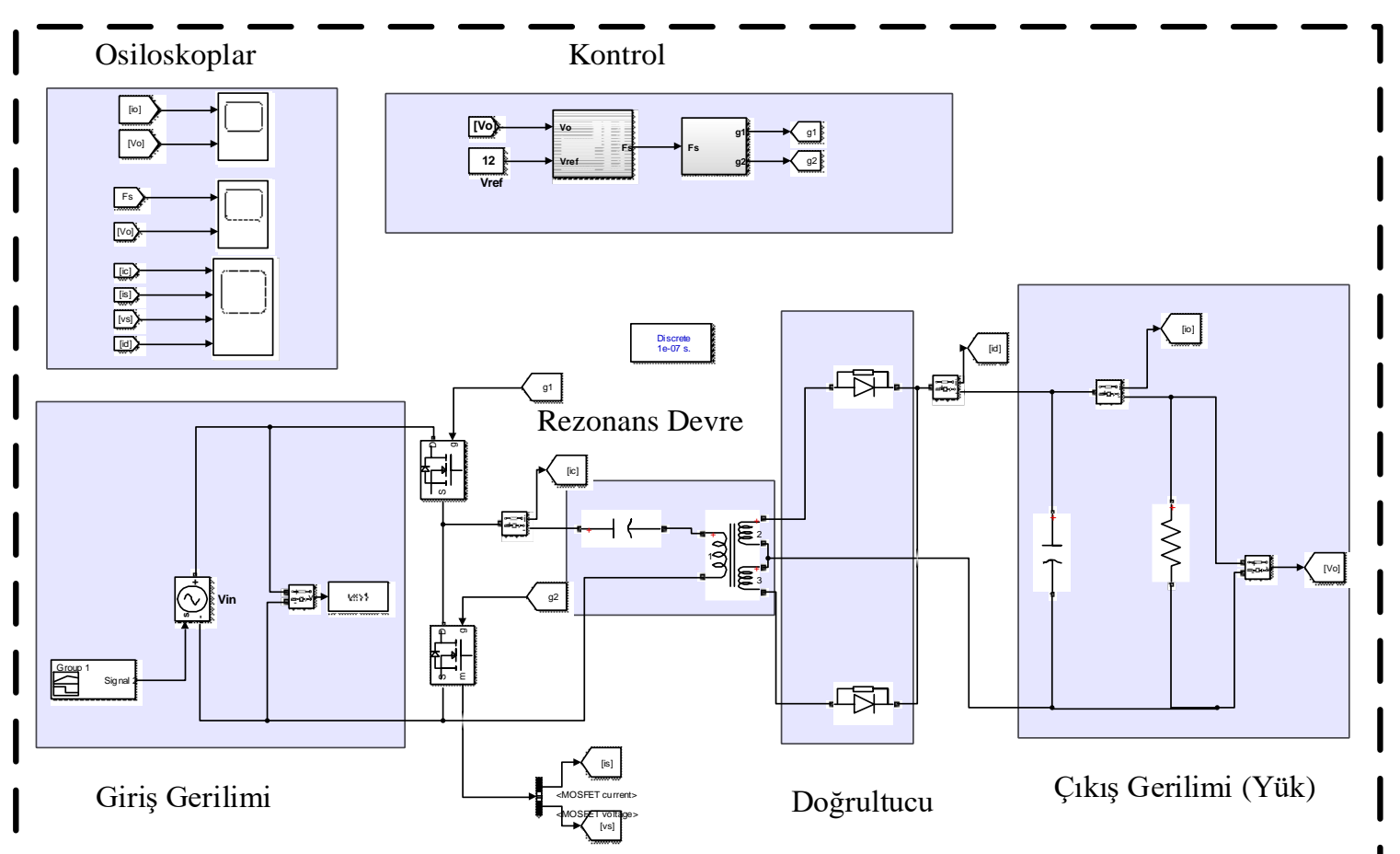

Şekil 4. Rezonans dönüştürücü benzetim ortamındaki kurulumu (Installation in resonant transducer simulation environment)

\section{BULGULAR VE ANALIZ (FINDINGS AND ANALYSIS)}

LLC Rezonans dönüştürücünün 4 farklı yük durumunda $(\% 100, \% 75, \% 50, \% 25)$ çıkış gerilimi, referans sinyali ve yük akımı Şekil 5 te gösterilmiştir. Çıkış gerilim dalgalanması $0.1 \mathrm{~V}$ $(\leq 1 \%)$ tan küçük olduğu şekilde görülmektedir.

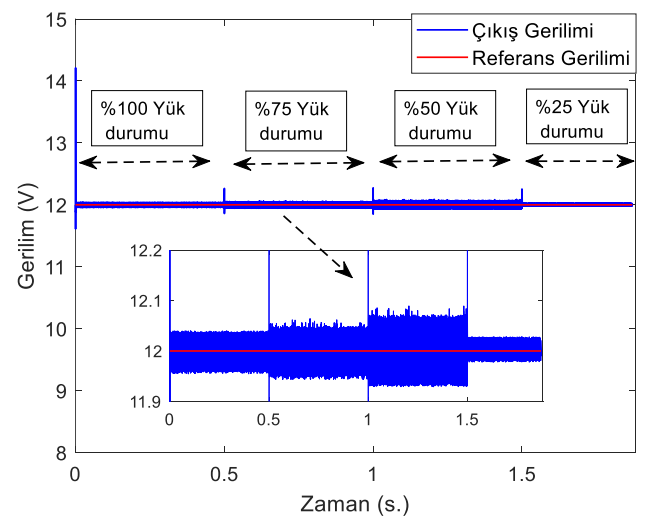

(a)
Batarya tam yük durumunda $25 \mathrm{~A}$ akım çekmesine rağmen $\% 25$ yük durumunda ise $5 \mathrm{~A}$ akım çekmektedir. Değişen yük durumunda Sabit gerilimde şarj işlemi gerçekleştirebilmek için PI tabanlı kontrol yöntemi ile gerilim $12 \mathrm{~V}$ ta sabit tutulmuşstur.

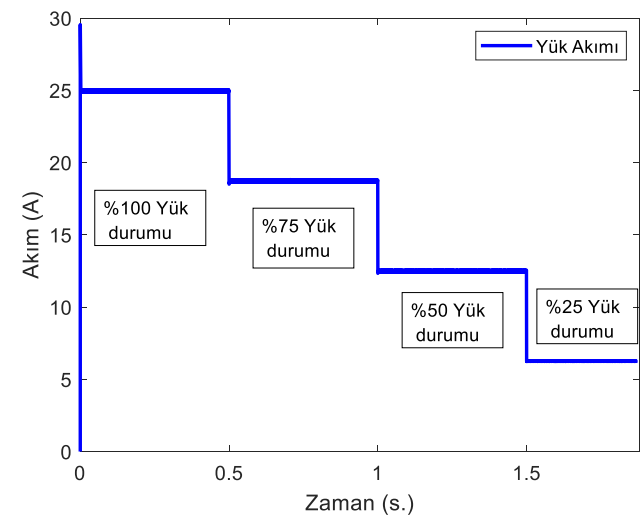

(b)

Şekil 5. (a) Çıkış gerilimi ve referans sinyali, (b)Yük akımı ((a) Output voltage and reference signal, (b) Load current)

Şekil 6 da rezonans kapasitör akımı trafonun manyetik bobin akımı, anahtar akımı (Mosfet1) Anahtar gerilimi (Mosfet1) ve diyot akımı detaylıca gösterilmiş̧ir. Rezonans kapasitör akımı ve manyetik bobin akımı incelendiğinde rezonans noktaları tespit edilebilmektedir. 


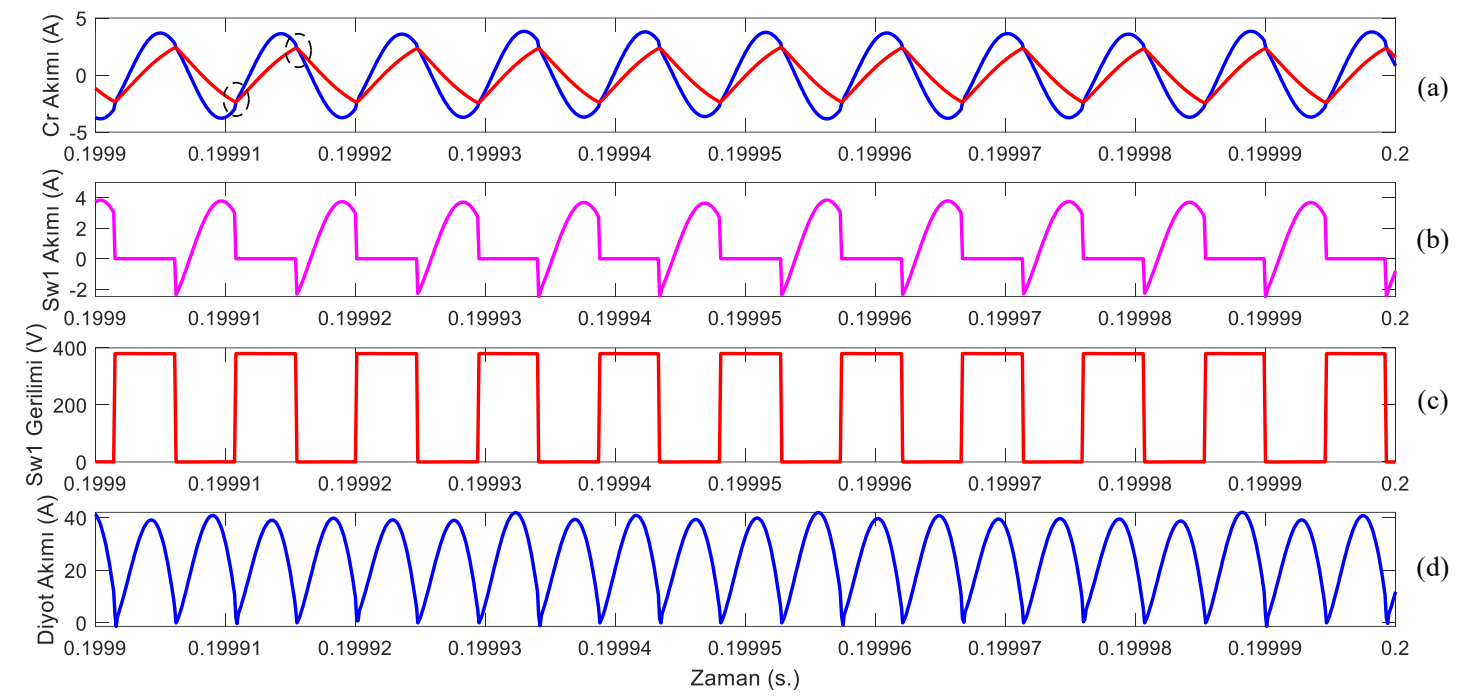

Şekil 6. LLC Rezonans dönüştürücü elektriksel karakteristikleri, (a) Rezonans kapasitör akımı, (b) Anahtar (Mosfet) akımı, (c) Anahtar (Mosfet) gerilimi, (d) diyot akımı (LLC Resonant converter electrical characteristics, (a) Resonant capacitor current, (b) Switch (Mosfet) current, (c) Switch (Mosfet) voltage, (d) diode current)

Anahtarlama frekansı rezonans dönüştürücü kontrolünde önemli bir parametre olduğundan dolayı değişen yük durumuna göre kontrol metodunun anahtarlama frekansına etkisi Şekil 7 de gösterilmiştir. Yük durumlarının değişmesinden dolayı anahtarlama frekansı PI tabanlı kontrol yöntemi sayesinde düzenlenmektedir. Böylece yük akımı değişmesine rağmen çıkış gerilimi sabit tutulabilmektedir.
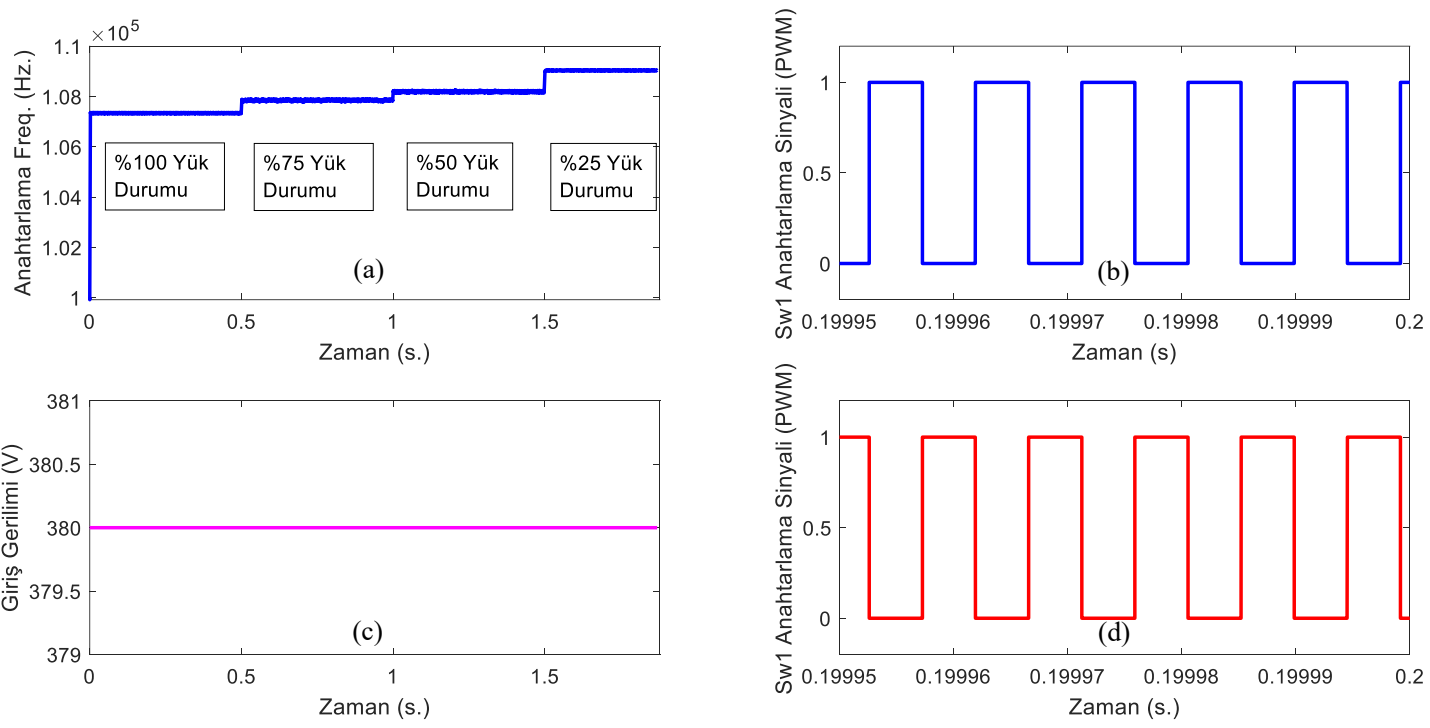

Şekil 7. (a) Anahtarlama Frekansı (b) Anahtarlama Sinyali (Mosfet1) (c) Anahtarlama, Giriş Gerilimi (d) Anahtarlama Sinyali (Mosfet2) ((a) Switching Frequency, (b) Switching Signal (Mosfet1), (c) Switching, Input Voltage, (d) Switching Signal (Mosfet2))

Şebeke geriliminde olası ani değişiklikler DClink geriliminde düzensizlik oluşturabilir. $\mathrm{Bu}$ durumda DC-link kontrolü sağlanana kadar LLC rezonans dönüştürücünün giriş gerilimi anlık olarak değişebilir, bu durumu test etmek amacı ile LLC rezonans dönüştürücünün giriş gerilimi anlık $380 \mathrm{~V}$ -
$400 \mathrm{~V}$ arasında değiştirilerek PI kontrolün davranış1 gözlemlenmiş ve PI kontrolün dinamik tepkisi Şekil 8 de gösterilmiştir. Ani giriş gerilimi değişikliğine rağmen PI kontrol yöntemi sayesinde çıkış gerilimi sabit tutularak bataryanın sabit gerilimde şarj edilebileceği gözlemlenmiştir. 


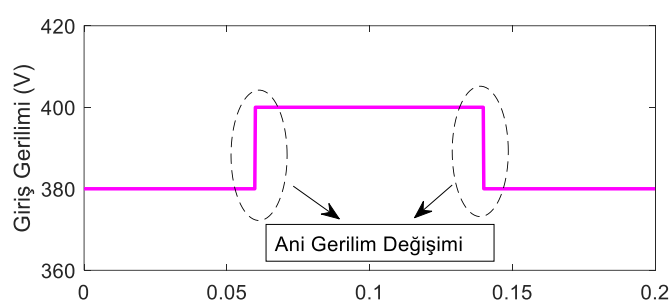

(a)

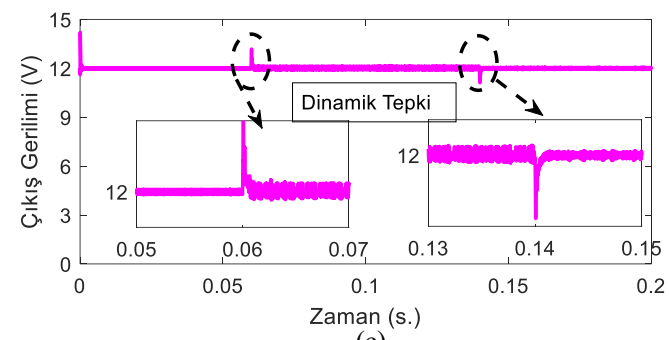

(c)

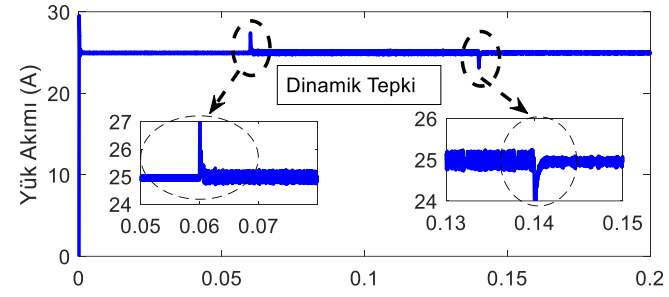

(b)

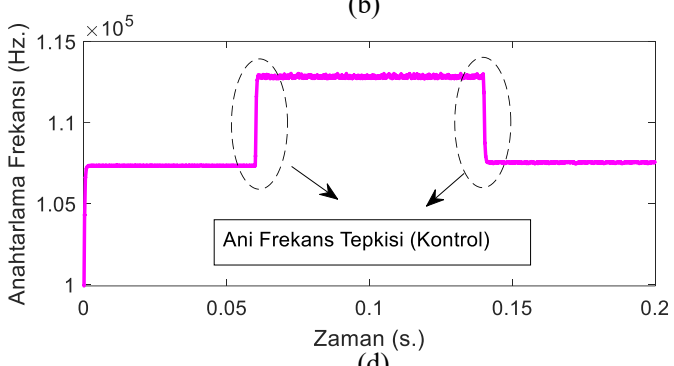

(d)

Şekil 8. Giriş gerilimi değişiminin yarım köprü LLC rezonans dönüştürücü üzerindeki etkisi. (a) Giriş Gerilimi,

(b) Çıkış akımı, (c) Çıkış Gerilimi, (d) Anahtarlama Frekansı (Effect of input voltage variation on half-bridge LLC resonant converter. (a) Input Voltage, (b) Output current, (c) Output Voltage, (d) Switching Frequency)

\section{SONUÇ (RESULTS)}

$\mathrm{Bu}$ çalışmada Elektrikli araçlarda batarya şarj ünitesi için kullanılabilen izolasyonlu yarım köprü LLC rezonans dönüştürücü farklı yük durumları ve ani giriş gerilimi değişimlerinde test edilip sonuçlar alınmıştır. Giriş gerilimi $380 \mathrm{~V}$ olan ve çıkış gerilimi 12 V LLC rezonans dönüştürücü Matlab/Simulink ortamında tasarlanmış olup ayrıca laboratuvar ortamında kurulumu gerçekleştirilmiştir. Laboratuvar ortamı için Texas instrument 'Resonant LLC Half-Bridge DC/DC Converter' kiti kullanılmıştır.

Analiz çalışmasını detaylandırmak için girişte ani değişen gerilim verilmiş ve PI tabanlı kontrol yönteminin performansı Şekil 8'de gösterilmiştir. Ayrıca farklı yük koşullarında (\%100, \%75, \%50, $\% 25$ ) test edilen LLC rezonans dönüştürücünün çıkış gerilimi, yük akımı ve frekans tepkisi Şekil 5 te detaylandırılmıştır. Analizi yapılan devrenin çıkış gerilim dalgalanması $0.1 \mathrm{~V} \quad(\leq \% 1)$ civarında ölçülmüştür. Sonuç olarak yalıtımlı yarım köprü LLC rezonans dönüştürücü modelinin; yüksek güç yoğunluğu, düşük anahtarlama kayıpları ve aynı zamanda tasarım yapılırken çıkış filtresine gereksinim duyulmaması gibi önemli avantajlara sahip olduklarından dolayı elektrikli araçların batarya şarj ünitesinde kullanılan dc-dc dönüştürücüler arasında ciddi adaylardan biri olarak düşünülebilir.

\section{TEŞEKKÜR (ACKNOWLEDGEMENT)}

$\mathrm{Bu}$ çalışma Harran Üniversitesi Bilimsel Araştırma Projeleri Birimi tarafından desteklenmiştir. Proje Numarası: 19012. Calıșma labaratuvarının düzenlenmesinde yardımcı olan Serkan KAHRAMAN'a teșekkür ederiz.

\section{ÇIKAR ÇATIŞMASI BÍLDİRIMİ (CONFLICT OF INTEREST STATEMENT)}

Yazarlar tarafından herhangi bir çıkar çatışması bildirilmemiştir.

\section{KAYNAKLAR (REFERENCES)}

[1] G. Waltrich and M.L. Heldwein, "Modeling and simulation of electric vehicles (EVs) and design of batteries for EVs," IEEE Southern Power Conference, 2015, November 29th to December 2nd, 2015.

[2] C-Y. Oh, D-H. Kim, D-G. Woo, W-Y. Sung and B-K Lee, "A High-Efficient Non isolated Single Stage On-Board Battery Charger for Electric Vehicles," IEEE Transactions On Power Electronics, vol. 28, no. 12, pp. 5746-5757, 2013.

[3] S. Li, J. Deng and C.C. Mi, "Single-Stage Resonant Battery Charger with Inherent Power Factor Correction for Electric Vehicles," IEEE Transactions On Vehicular Technology, vol. 62, no. 9, pp. 4336-4344, 2013.

[4] S. Dusmez and A. Khaligh, "A ChargeNonlinear-Carrier-Controlled Reduced-Part SingleStage Integrated Power Electronics Interface for 
Automotive Applications," IEEE Transactions On Vehicular Technology, vol. 63, no. 3, pp. 1091-1103, 2014.

[5] B. Koushki, A. Safaee, P. Jain and A. Bakhshai, "Review and Comparison of Bi-Directional AC-DC Converters with V2G Capability for On-Board EV and HEV," IEEE Transportation Electrification Conference and Expo (ITEC) 2014, pp. 1-6.

[6] U.R. Prasanna, A.K. Singh and K. Rajashekara, "Novel Bidirectional Single-phase Single-Stage Isolated AC-DC Converter with PFC for Charging of Electric Vehicles," IEEE Transactions On Transportation Electrification, vol. 3, no. 3, pp. 536544, 2017.

[7] H.S. Ribeiro and B.V. Borges, "HighPerformance Voltage-Fed AC-DC Full-Bridge Single-Stage Power Factor Correctors with a Reduced DC Bus Capacitor," IEEE Transactions On Power Electronics, vol. 29, no. 6, pp. 2680-2692, 2014.

[8] C.A. Cheng, C.H. Chang, T.Y. Chung and F.L. Yang, "Design and Implementation of a SingleStage Driver for Supplying an LED Street-Lighting Module with Power Factor Corrections, " IEEE Transactions On Power Electronics, vol. 30, no. 2, pp. 956-966, 2015.

[9] M. Kwon, S. Choi, "An Electrolytic Capacitorless Bidirectional EV Charger for V2G and V2H Applications," IEEE Transactions On Power Electronics, vol. 32, no. 9, pp. 6792-6799, 2013.

[10] U. Yilmaz, O. Turksoy and A. Teke, "Intelligent control of high energy efficient two-stage battery charger topology for electric vehicles," Energy, vol.186, 115825, 2019.

[11] U. Yilmaz, O. Turksoy and A. Teke, "Improving a battery charger architecture for electric vehicles with photovoltaic system," J Energy Res., vol. 44, pp.4376-4394, 2020. doi: 10.1002/er.5211

[12] S. Alqahtani, S. Ganesan and M. A. Zohdy, "The Comparison between PI and PID Controllers in Engine Speed Control Model," 2020 IEEE International Conference on Electro Information Technology (EIT), 2020, pp. 629-634. doi: 10.1109/EIT48999.2020.9208313.

[13] J. Deng, S. Li, S. Hu, C. C. Mi and R. Ma, "Design Methodology of LLC Resonant Converters for Electric Vehicle Battery Chargers," in IEEE Transactions on Vehicular Technology, vol. 63, no. 4, pp. 1581-1592, May 2014. doi: 10.1109/TVT.2013.2287379.

[14] N. Shafiei and M. Ordonez, "Improving the Regulation Range of EV Battery Chargers with L3C2 Resonant Converters," in IEEE Transactions on Power Electronics, vol. 30, no. 6, pp. 3166-3184, June 2015. doi: 10.1109/TPEL.2014.2336668.

[15] C.C Hua, Y.H Fang and C.W Lin, "LLC resonant converter for electric vehicle battery chargers," IET Power Electron., , Vol. 9, no. 12, pp. 2369-2376, 2016.

[16] D.H. Kim, M.S. Kim, S.H. Nengroo, C.H. Kim and H.J. Kim, "LLC Resonant Converter for LEV (Light Electric Vehicle) Fast Chargers," Electronics, vol. 8, no. 362, pp. 1-14, 2019.

[17] M. Li, Q. Chen, X. Ren, Y. Zhang, K. Jin and B. Chen, "The integrated LLC resonant converter using center-tapped transformer for on-board EV charger," 2015 IEEE Energy Conversion Congress and Exposition (ECCE), Montreal, QC, Canada, 2015, pp. 6293-6298.

doi: 10.1109/ECCE.2015.7310542.

[18] X. Dan Gumera, A. Caberos and S. Huang, "Design and Implementation of a High Efficiency Cost Effective EV Charger Using LLC Resonant Converter," 2017 Asian Conference on Energy, Power and Transportation Electrification (ACEPT), Singapore, 2017, pp. 1-6.

doi: 10.1109/ACEPT.2017.8168618.

[19] S. Kimura, K. Nanamori, M. Noah and M. Yamamoto, "A novel llc resonant dc-dc converter with integrated transformer," 2017 IEEE International Telecommunications Energy Conference (INTELEC), Broadbeach, QLD, Australia, 2017, pp. 506-510.

doi: 10.1109/INTLEC.2017.8214186.

[20] Y. Daş, A. Tekin, M. Boztepe and C. Tarhan, "A Robust Voltage Control Method for Universal EV Battery Charger LLC Resonant Converters," 2019 IEEE International Conference on Sustainable Energy Technologies and Systems (ICSETS), Bhubaneswar, India, 2019, pp. 120-125. doi: 10.1109/ICSETS.2019.8744865.

[21] H. Bai, D. Yang, J. Song, Q. Su, B. Duan and C. Zhang, "Linear Active Disturbance Rejection Control of LLC Resonant Converters for EV Chargers," 2020 Chinese Automation Congress (CAC), Shanghai, China, 2020, pp. 993-998. doi: 10.1109/CAC51589.2020.9327865. 
[22] J.J. Yong, V.K. Ramachandaramurthy, K.M. Tan and N. Mithulananthan, "A review on the stateof-the-art technologies of electric vehicle, its impacts and prospects," Renewable and Sustainable Energy Reviews, vol. 49, pp. 365-385, 2015.

[23] Designing an LLC Resonant Half-Bridge Power Converter. 2010 Texas Instruments Power Supply Design Seminar. pp. 1-27.

[24] R.L. Lin and L.H. Huang, "Efficiency Improvement on LLC Resonant Converter Using Integrated LCLC Resonant Transformer," IEEE Transactions On Industry Applications, vol. 54, no. 2, pp.1756-1764, 2018.

[25] H.N. Vu and W. Choi, "A Novel Dual FullBridge LLC Resonant Converter for $\mathrm{CC}$ and $\mathrm{CV}$ Charges of Batteries for Electric Vehicles". IEEE Transactions On Industrial Electronics, vol. 65, no. 3, pp. 2212-2225, 2018.
[26] M. Noah, S. Endo, H. Ishibashi, K. Nanamori J. Imaoka, K. Umetani and M. Yamamoto, "A Current Sharing Method Utilizing Single Balancing Transformer for a Multiphase LLC Resonant Converter with Integrated Magnetics," IEEE Journal of Emerging and Selected Topics in Power Electronics, vol. 6, no. 2, pp. 977-992, 2017.

This is an open access article under the CC-BY license (https://creativecommons.org/licenses/by/4.0/)

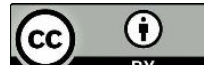

